\title{
ThyPROgr: Translation and validation in Greek of the thyroid-specific quality of life questionnaire ThyPRO
}

Gesthimani Mintziori' ${ }^{12}$, Torquil Watt ${ }^{3}$, Dimitrios Pournaras ${ }^{1}$, Tzortzis Emmanouil' ${ }^{1}$, Marina Kita ${ }^{2}$, Dimitrios Goulis ${ }^{1}$

1. Unit of Reproductive Endocrinology, First Department of Obstetrics and Gynecology, Aristotle University Medical School, Thessaloniki, Greece

2. Department of Endocrinology, Ippokratio General Hospital, Thessaloniki, Greece

3. Department of Medical Endocrinology, Copenhagen University Hospital Rigshospitalet, Denmark

\section{Introduction}

Assessing the quality of life of patients with thyroid disease does represent a challenge.

ThypRO questionnaire has been developed recently and comprises a reliable and valid scale structure of a patient-reported outcome measuring thyroid-specific quality of life (1) (2).

The aim of the current study was the translation and the validation in Greek language of the ThyPRO questionnaire.
- After formation, ThyPROgr was tested among five patients with thyroid disorders with cognitive interview techniques and additional changes in wording were made, when necessary.

\section{Results}

ThyPRO was translated and validated in Greek according to standard methodology for translation of patient-reported outcomes.

ThyPROgr comprises of 85 questions, organized in 13 sections.

\section{Material and Methods}

The standard methodology for translation of patient-reported outcomes was followed.

- Two independent translators, Greek native speakers, fluent in english, were asked to translate THyPRO questionnaire from English to Greek, in order to form a consensus version of the questionnaire, after comparing the two versions.

- A third translator was asked to back-translate this consensus version to English.

- An in-country consultant and the developer of ThyPRO questionnaire then reviewed the backwards translation and additional revisions were made.

\section{Conclusion}

ThyPROgr provides the possibility to measure patient-reported outcomes regarding thyroidspecific quality of life in the Greek population.

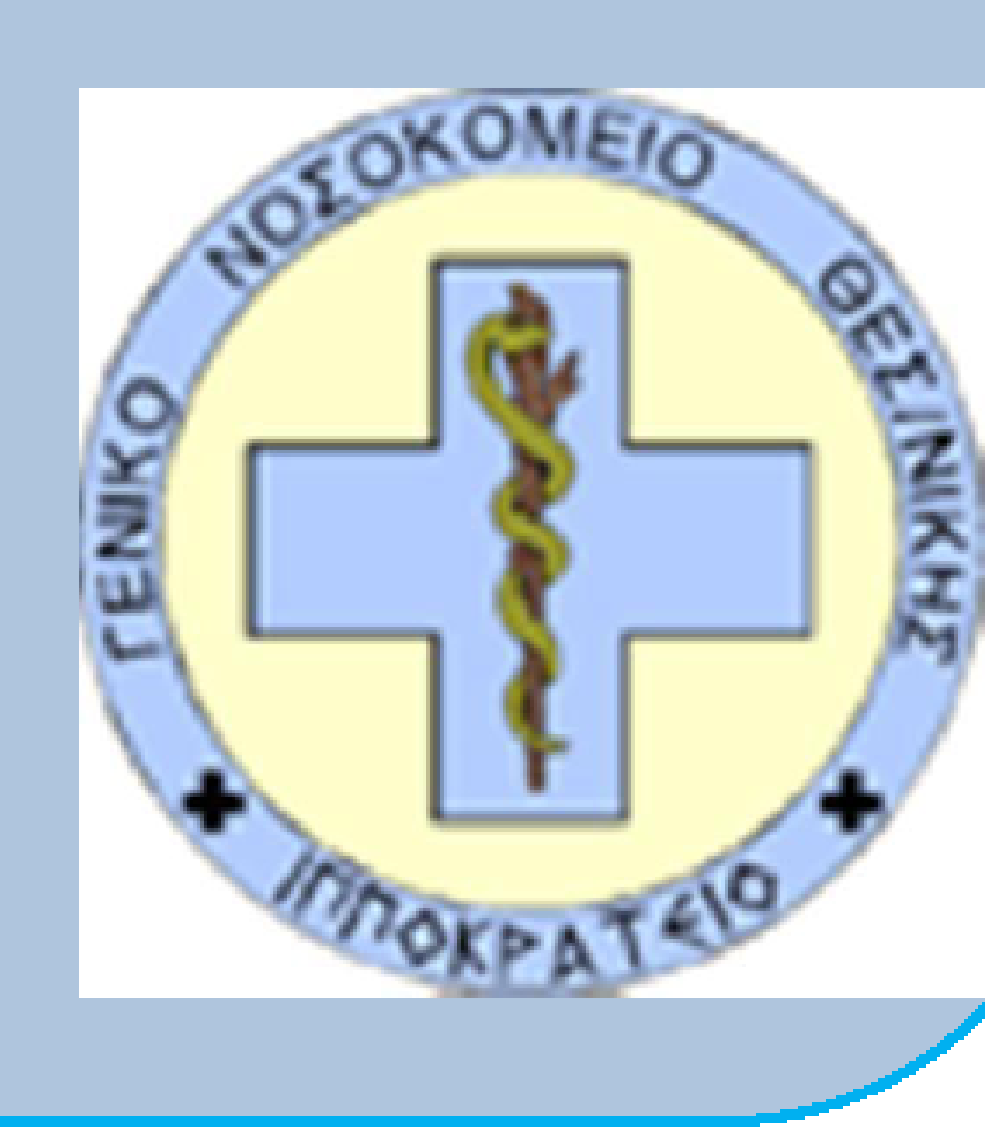

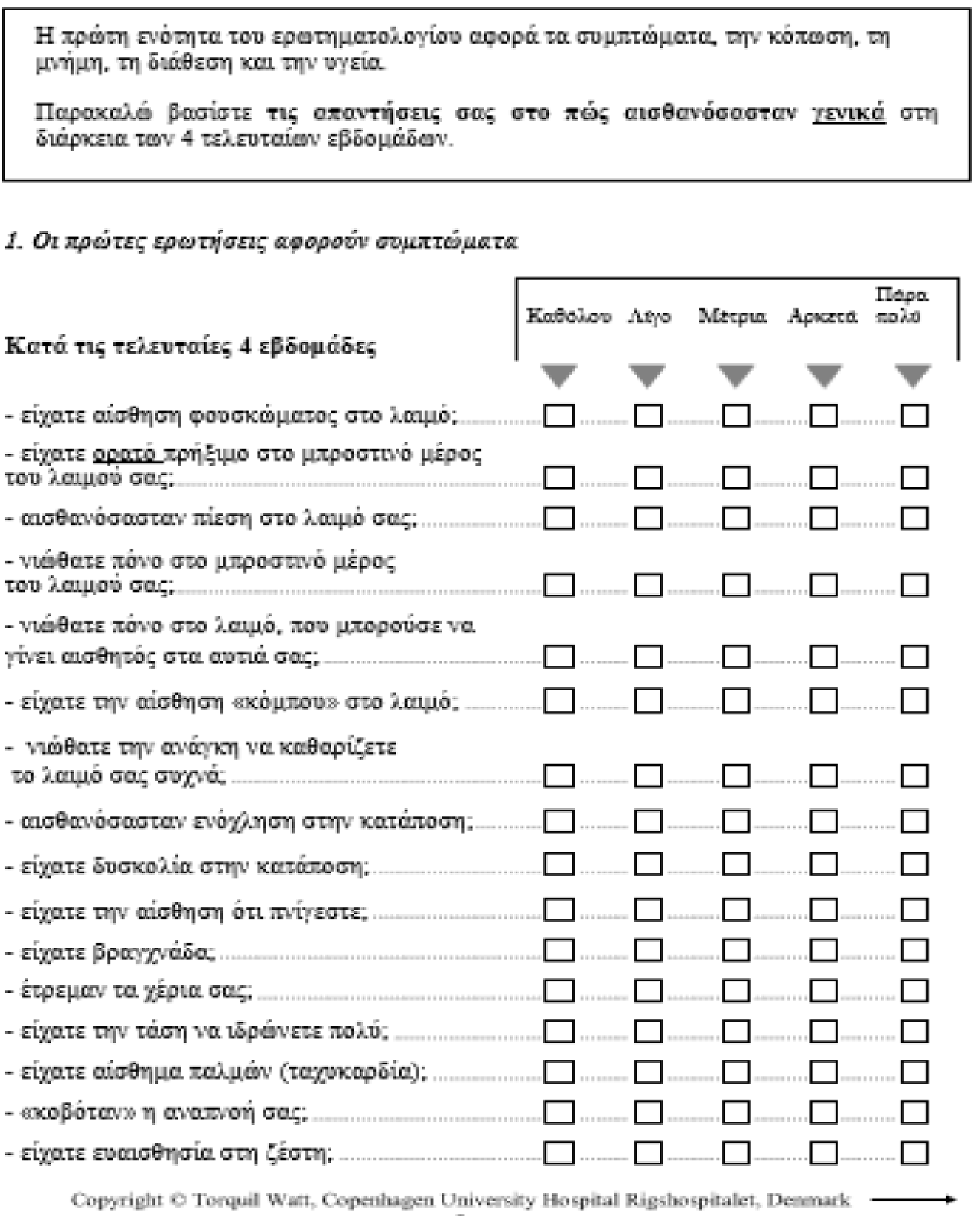

Fig 1. The first page of ThyPROgr

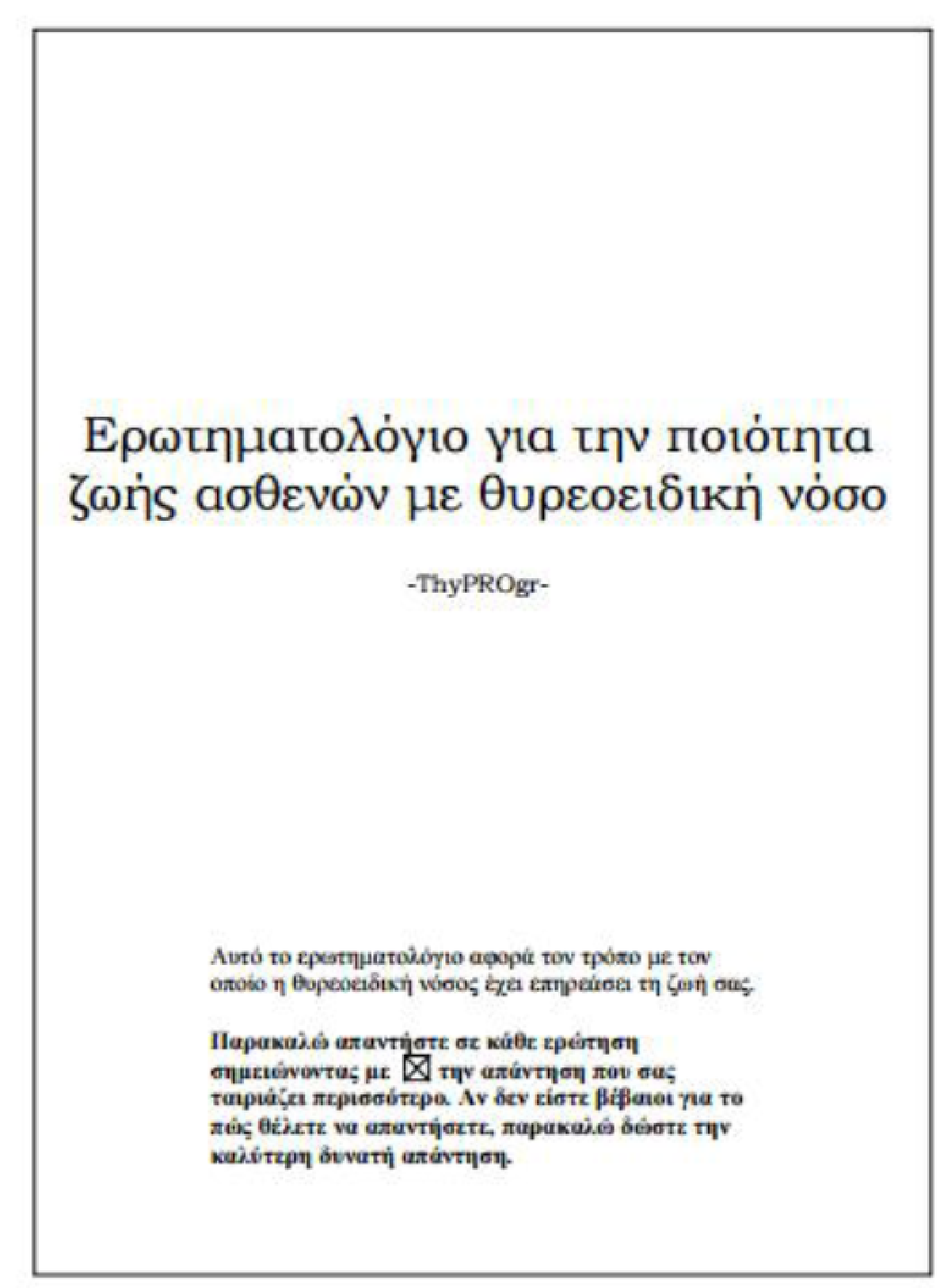

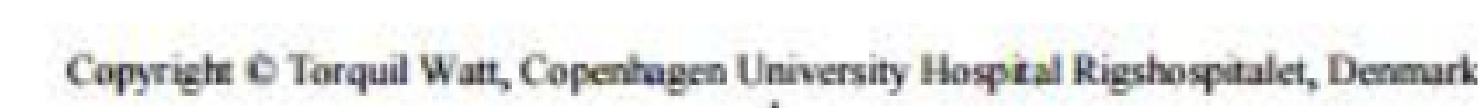

\section{References}

1. Watt $T$, Hegedüs $L$, Groenvold M, Bjorner JB, Rasmussen AK, Bonnema SJ, Feldt-Rasmussen $U$. Validity and reliability of the novel thyroid-specific quality of life questionnaire, ThyPRO. Eur J Endocrinol 2010;162(1):161-7

2. Wong CK, Lang BH, Lam CL. A systematic review of quality of thyroid-specific health-related quality of life instruments recommends ThyPRO for patients with benign thyroid diseases. J Clin Epidemiol. 2016 (in press) 